\title{
Perceptual components of situation models
}

\author{
REBECCA FINCHER-KIEFER \\ Gettysburg College, Gettysburg, Pennsylvania
}

\begin{abstract}
These experiments examined the hypothesis that situation model construction involves perceptual processing-specifically, processing that involves visuospatial information. In this research, a dualtask paradigm was used to demonstrate that tasks that engage visuospatial processes interfere more with the generation of a situation model than tasks that are less likely to involve these processes or tasks that are verbal in nature. Using Albrecht and O'Brien's (1993) contradiction effect as evidence of situation model construction, Experiment 1 demonstrated that participants reading short texts while simultaneously holding high-imagery sentences in memory failed to show a significant contradiction effect in comparison with readers holding low-imagery sentences in memory. In Experiment 2, participants reading texts while retaining a difficult visuospatial memory load showed disrupted comprehension in comparison with readers retaining a verbal memory load.
\end{abstract}

Many reading researchers have explored the processes used in situation model construction (e.g., Kintsch \& Welsch, 1991; Myers \& O'Brien, 1998; Zwaan, Langston, \& Graesser, 1995), but there has been less investigation into the exact nature of situation models. The traditional theoretical position has been that situation models are sets of propositions and therefore "amodal" symbolic systems (Kintsch, 1998; van Dijk \& Kintsch, 1983). Recently, theorists have suggested that situation model construction may involve "modal" symbol systems - that is, perceptual symbols that represent knowledge (Barsalou, 1999; Glenberg, 1997; Glenberg \& Robertson, 1999; MacWhinney, 1999; Zwaan, 1999). In order to account for the phenomenological richness of discourse comprehension, the perceptual symbols framework suggests that comprehension entails establishing an analogue relationship between the text and the reader's background knowledge. Barsalou (1999) claims that knowledge may take the form of perceptual symbols, which are derived directly from perceptual experiences. The use of these perceptual symbols in reading facilitates the construction of a situation model (Glenberg \& Robertson, 1999). As Zwaan (1999) argues, amodal and modal systems may not be mutually exclusive; readers may use both types of representation in reading comprehension. However, the recent theoretical claims put forth by Barsalou (1999), Glenberg (1997), and MacWhinney (1999) suggest that "the time is

This research was supported by a Research and Professional Development Grant from Gettysburg College. These experiments were presented in poster form at the 39th annual meeting of the Psychonomic Society in November 1998. I thank Paul R. D'Agostino for his help and guidance with this research program and his comments on this manuscript. I am grateful to Ed O'Brien and Arnold Glass for providing their text materials, and to Shauna Landis, Amanda Gingerich, and Sara Abbot for their help in conducting this research. Correspondence concerning this article should be addressed to R. Fincher-Kiefer, Department of Psychology, Gettysburg College, Gettysburg,PA 17325 (e-mail: rfincher@ gettysburg.edu). ripe for investigationsinto the role of perceptual representations in discourse comprehension"(Zwaan, 1999, p. 87).

The typical approach for investigating how readers represent perceptual information-specifically, visual or spatial information- has been to give texts that elicit a visual representation analogous to the three-dimensional space of our real experiences (see Morrow, 1994, for a review of this research) or to give texts that are accompanied by a photograph (Glenberg \& Langston, 1992; Glenberg \& Robertson, 1999). The intent of the present research was to examine situation models derived from texts that do not describe spatial relations or are not accompanied by a picture. This research explores the possibility that even situation models generated from nonspatial texts may have perceptual properties similar to perceptual experience. In the present experiments, I used texts that did not inherently require or elicit a spatial representation; these were texts that described everyday actions and events, such as ordering food at a restaurant. Experiment 1 tested the hypothesis that the generation of situation models engages perceptual processing that involves visuospatial information. In order to explore this issue, I borrowed the dual-task logic used in some cognitiveperceptual research (Baddeley, 1992; Brooks, 1968). When readers are comprehending a text, if given a concurrent task that engages visuospatial processes, this should impair situation model construction if it, too, engages the same processes. In Experiment 1, I gave readers sets of either high-or low-imagery sentences to hold in memory while reading texts. The hypothesis tested was that holding high-imagery sentences in memory would involve some of the processes used in situation model construction. Therefore, readers should have more difficulty generating a situation model when holding high-imagery sentences in memory than when holding low-imagery sentences in memory.

In order to examine the impact of this memory load on situation model construction, I used O'Brien and Albrecht's 
(1992; Albrecht \& O'Brien, 1993) measure of situation model construction, the contradiction effect. This effect occurs when readers are given a passage to read and, toward the end, encounter a critical sentence that is locally coherent with the preceding sentence but is either consistent or inconsistent with information presented earlier in the passage. Here is an example: Mary may be either initially described as a vegetarian or as someone who enjoys eating at McDonald's often. This is then followed by several sentences that are intended to background that information so that no further mention is made of Mary's eating habits. The critical sentence follows then and states that Mary ordered a hamburger. Using texts such as this, O'Brien and his colleagues (Albrecht \& O'Brien, 1993; Huitema, Dopkins, Klin, \& Myers, 1993; Myers, O’Brien, Albrecht, \& Mason, 1994) have found that reading times for the critical sentence were significantly longer in the inconsistent text condition than in the consistent text condition. This is a situation model effect, because it demonstrates that readers are engaged in maintaining global coherence even when the text is locally coherent. The contradiction effect provides a measure of situation model construction - if a reader fails to establish a global understanding of the situation described by the text, the contradiction effect would not occur.

In Experiment 1, I predicted that the maintenance of high-imagery sentences and the generation of a perceptuallike situation model would engage the same processes, causing interference in one or both of these tasks. In the dual-task paradigm, impairment on either task is evidence that the two tasks share resources (Kruley, Sciama, \& Glenberg, 1994; Sims \& Hegarty, 1997). Participants in Experiment 1 were told that their primary task was to remember sets of sentences presented to them. They were also told that they would be reading short texts between when they were given the sets of sentences and when they would have to recall them. Given the emphasis on the memory task, I expected that the interference from shared processes and resources would be reflected in impairment on the comprehension task. Therefore, the primary prediction was that readers holding high-imagery sentences in memory would show a reduced contradiction effect in comparison with readers holding low-imagery sentences in memory. However, if construction of the situation model competes for the processes used in holding high-imagery sentences in memory, interference could also be reflected in overall comprehension difficulty for the texts read by the high-imagery participants (longer reading times for all the sentences of the texts) or in poor memory for the high-imagery sentences.

\section{EXPERIMENT 1}

\section{Method}

Participants. One hundred students from Gettysburg College took part in this experiment. Three students' data were not included in the analyses because they answered more than half of the comprehension questions that followed each text incorrectly. Of the re- maining 97 participants, 48 were in the high-imagery condition and 49 were in the low-imagery condition. Participants either received credit for their research participation requirement in their general psychology course or were paid $\$ 5.00$ for their participation.

Design and Materials. This study used a 2 (type of memory load: high-imagery sentences vs. low-imagery sentences) $\times 2$ (sentence type: consistent sentences vs. inconsistent sentences) mixed design. Type of memory load was manipulated between subjects, and sentence type was manipulated within subjects. Four dependent variables were computed: reading time for the critical sentence, reading time for the sentence that followed the critical sentence, average reading times for the sentences that preceded the critical sentences, and percentage correct for the memory load task.

Fifteen sets of five high- and five low-imagery sentences were chosen from materials used by Glass and his colleagues (e.g., Glass, Eddy, \& Schwanenflugel, 1980; Glass, Millen, Beck, \& Eddy, 1985). An example of a high-imagery sentences is A star of David has six points; an example of a low-imagery sentence is The shortest month is February. In Glass's research, these sentences had been rated on imagery as well as equated on truth agreement, comprehensibility ratings, and word length.

Fifteen experimental passages were chosen from the materials used by Albrecht and O'Brien (1993). A full description of these passages can be found in Albrecht and O'Brien, and an example passage is presented in the Appendix. Fourteen experimental passages were randomly divided into two sets of 7 passages, and 1 passage was chosen to be used as a practice passage. Sentence type was counterbalanced across participants so that half of the participants received one set of 7 passages in the consistent condition and the other set in the inconsistent condition, whereas the other half of the participants received the reverse. The order of the 14 passages was randomly determined but was the same for each participant. A simple question followed each passage to ensure that the reader was comprehending each passage.

Procedure. The participants were randomly assigned to one of two memory load conditions and one of two material sets. Each participant was individually tested in a session that lasted approximately $40 \mathrm{~min}$. All of the materials were presented on a video monitor controlled by an IBM-PC 486 computer.

Participants were told that they were taking part in a memory experiment and would be asked to remember sets of five sentences. Each trial started with the word Ready, presented for $2 \mathrm{sec}$ at the center of the computer screen. This was followed by the first sentence of the memory set, which was on the screen for $6 \mathrm{sec}$. The participants read the sentence aloud to the experimenter and were told to try to understand the sentence because they would be asked to remember it. This procedure continued until the participants had been presented five sentences. After this memory set, participants were given a "distractor" task, which was to read a short text presented on the computer screen one sentence at a time. A string of asterisks appeared in the center of the screen for $2 \mathrm{sec}$, signaling that the text was to begin. These asterisks were replaced by the first sentence of the text. The participants read each sentence of the text silently at their own normal reading pace. When they were ready for the next sentence, they pressed the space bar. Each press of the space bar erased the current sentence and presented the next sentence. Reading time was the time between keypresses. When the participant pressed the space bar at the end of the passage, the word Question appeared at the center of the screen for $2 \mathrm{sec}$. This was followed by the comprehension question. In response to this question, the participants pressed either the Yes or the No key (the "B" key and the "N" key, respectively, covered with a "Y" and "N" sticker). If they responded incorrectly to this question, the word ERROR was presented at the center of the screen for $2 \mathrm{sec}$.

Immediately after the subject had responded to this question, the words Memory Test appeared in the center of the screen. The participants recalled as many of the five sentences presented before the 
text as possible or recalled any part of the sentences. The experimenter had a list of the sentences to be recalled and recorded each participant's recall by underlining what part of each sentence was remembered. When the participant had recalled as much as he/she could from the memory set, the experimenter started this same cycle again. This procedure continued until all 14 of the experimental passages had been read. Participants received one practice session.

\section{Results}

Reading times. The reading times for all sentences of the texts were recorded, with the reading times of the critical target sentences and the posttarget sentences of primary interest. The analyses for the posttarget sentences showed few significant differences across conditions, and when there were differences, they were in the same direction as were the effects for the critical target sentences. Therefore, only the analyses for the critical target sentences will be presented. Reading times for critical sentences that were three standard deviations from their mean were discarded. This eliminated less than $3 \%$ of the data. The reading time data were analyzed two ways: an analysis that included reading times for the texts in which participants had answered the comprehension question incorrectly (which occurred $11.7 \%$ of the time across all participants), and an analysis that excluded reading times for the texts in which the participants had made comprehension errors. There were no differences between these analyses, so only the results of the analyses for the reading times computed when there were no comprehension errors are presented. In both experiments reported in this article, I conducted analyses of variance (ANOVAs) on both subject $\left(F_{1}\right)$ and item $\left(F_{2}\right)$ means, and I used $p<.05$ unless otherwise noted.

The reading times for the critical target sentences are presented in Table 1. Critical sentences were read more slowly for participants in the high-imagery memory load condition than in the low-imagery memory load condition $\left[F_{1}(1,95)=6.52, M S_{\mathrm{e}}=40,667 ; F_{2}(1,26)=7.39\right.$, $\left.M S_{\mathrm{e}}=23,952\right]$. Furthermore, critical sentences were read more slowly in the inconsistent sentence type condition than in the consistent sentence type condition $\left[F_{1}(1,95)=\right.$ $\left.6.70, M S_{\mathrm{e}}=76,437 ; F_{2}(1,26)=5.49, M S_{\mathrm{e}}=65,570\right]$. Despite the fact that the contradiction effect (significant reading time difference between consistent and inconsistent sentence types) was twice the size for the low-imageryload participants $(137 \mathrm{msec})$ as for the high-imageryload participants $(69 \mathrm{msec})$, the interaction of sentence type and memory load was not significant $\left[F_{1}(1,95)=\right.$ $2.73, M S_{\mathrm{e}}=76,437, p>.10 ; F_{2}(1,26)=2.26, M S_{\mathrm{e}}=$

Table 1

Mean Reading Times (in Milliseconds) for Critical Sentences as a Function of Sentence Type and Memory: Experiment 1

Sentence Type

\begin{tabular}{|c|c|c|c|c|}
\hline \multirow[b]{3}{*}{ Memory Load } & \multicolumn{4}{|c|}{ Вепा:пе 1уре } \\
\hline & \multicolumn{2}{|c|}{ Consistent } & \multicolumn{2}{|c|}{ Inconsistent } \\
\hline & $M$ & $S D$ & $M$ & $S D$ \\
\hline High imagery & 1,744 & 523 & 1,813 & 511 \\
\hline Low imagery & 1,470 & 462 & 1,607 & 510 \\
\hline
\end{tabular}

Table 2

Mean Percentage Correct Recall as a Function of Sentence Type and Memory Load: Experiment 1

\begin{tabular}{|c|c|c|c|c|}
\hline \multirow[b]{3}{*}{ Memory Load } & \multicolumn{4}{|c|}{ Sentence Type } \\
\hline & \multicolumn{2}{|c|}{ Consistent } & \multicolumn{2}{|c|}{ Inconsistent } \\
\hline & $M$ & $S D$ & $M$ & $S D$ \\
\hline High imagery & 58 & 14 & 57 & 14 \\
\hline Low imagery & 55 & 14 & 53 & 15 \\
\hline
\end{tabular}

$65,570, p>.10]$. However, planned comparisons confirmed the prediction that although the high-imagery memory load participants did not show the significant contradiction effect in their reading times $\left[F_{1}(1,47)=\right.$ $\left.1.25, p>.25 ; F_{2}(1,13)=2.56, p>.10\right]$, the low-imageryload participants did show the expected contradiction effect $\left[F_{1}(1,48)=7.41, M S_{\mathrm{e}}=61,656 ; F_{2}(1,13)=3.03\right.$, $\left.M S_{\mathrm{e}}=49,358, p=.07\right]$.

In order to determine whether there was a difference between the high-imagery memory load participants and the low-imagery memory load participants in overall comprehension difficulty, an analysis was conducted on average reading times for each participant for the sentences of the texts that preceded the critical sentences. In this analysis, the memory load variable but not the sentence type variable was analyzed, because the consistent/inconsistent manipulation was only relevant for the critical sentences. An analysis of these reading times indicated that the high-imagery memory load participants took significantly longer to read the texts $(M=2,825 \mathrm{msec})$ than the low-imagery memory load participants $(M=2,515$ msec) $\left[F_{1}(1,94)=4.10, M S_{\mathrm{e}}=51,851\right]$.

Recall. A strict scoring system was used for the recall data such that for each of the five sentences in each memory set, if all the words of a sentence were recalled, a score of 1 was given. If a participant did not remember all of the words but at least half of the words of a sentence were recalled, a score of .5 was received. The participant's final recall score was an average across all 14 memory sets.

The mean percentage correct recall for high- and lowimagery sentences, presented separately when they followed either consistent or inconsistent texts, is found in Table 2. These data indicate that high- and low-imagery sentences were recalled equally well $[F(1,95)=1.91, p>$ $.10]$, and recall of these sentences did not vary as a function of whether they were recalled following a critical sentence that was consistent or inconsistent with the earlier text $[F(1,95)=2.11, p>.10]$.

\section{Discussion}

Experiment 1 tested the hypothesis that holding highimagery sentences in working memory engaged the same perceptual processes that are needed for situation model construction. Therefore, I predicted that readers in the high-imagery memory load condition would show impaired situation model effects in comparison with readers in the low-imagery memory load condition. The results of this experiment supported this prediction. High-imagery 
memory load participants showed disrupted situation model construction in their reading time data-the absence of a significant contradiction effect-but lowimagery memory load participants showed the typical situation model effect. Furthermore, readers holding highimagery sentences in memory showed overall comprehension difficulty in their long reading times of all sentences of the text.

The reduction of the contradiction effect to $69 \mathrm{msec}$ for the high-imagery-load participants is quite remarkable, given that this effect, with the same materials, has been both reliable and robust in several published studies (e.g., Albrecht \& O'Brien, 1993; Huitema et al., 1993; Myers et al., 1994). One explanation for the loss of the contradiction effect is that for the high-imagery-load participants, holding these sentences required visuospatial resources needed for building a situation model-leading to long reading times and minimal evidence that the text was interpreted. An alternative explanation for these data is that high-imagery sentences were simply more difficult to maintain as a memory load than low-imagery sentences, and thus, the comprehension task suffered. However, the latter explanation was not supported by the recall data, which indicated that high-imagery sentences were not more difficult to recall; in fact, the trend in the data suggests that they were slightly easier to recall. This trend is certainly consistent with prior research comparing the recall of concrete as opposed to abstract material and high- as opposed to low-imagery materials (Glass et al., 1980; Paivio, 1969). I directly tested the issue of the difficulty of the memory load task in Experiment 2 and will therefore postpone further discussion of this alternative explanation until the discussion of that experiment.

The data from Experiment 1 suggest that the generation of a situation model and the maintenance of highimagery sentences in memory interfered with one another. In Experiment 2, I investigated this result further. If situation model construction engages perceptual processing that involves visuospatial information, then, when a reader must perform a concurrent task that is known to engage visuospatial processes, situation model effects should be disrupted. In previous research, the dual-task paradigm has been used to investigate when processes are shared between tasks (Baddeley, 1992). Recently, Sims and Hegarty (1997) employed a dual-task paradigm to demonstrate that a reasoning task that involved mental animation relied on the resources of working memory's visuospatial sketchpad. Kruley et al. (1994) employed a dual-task paradigm to show that the comprehension of text accompanied by a picture involves visuospatial working memory. Kruley et al. found a specific selective interference effect: The comprehension of texts in a picture condition but not in a no-picture condition interfered with a concurrent spatial task, the ability to remember the position of dots on a grid matrix. Furthermore, the presence of the picture with the text impaired the dot task but did not impair a concurrent nonspatial task, a task in which participants were asked to remember a string of digits while listening to the text. Kruley et al.'s interpretation of these results was that visuospatial working memory processes were needed to both hold the original dot matrix in memory and use the picture in comprehending the text (the picture did not interfere with the dot task in an experiment in which participants had no comprehension demands).

In Experiment 2, one group of participants held in memory an array of dots presented in a matrix and later decided whether a second matrix was the same as or different from the first. Another group of participants held in memory a string of letters and later decided whether a single letter presented to them was in that string. While participants were waiting for their second matrix/letter, they read short texts and answered simple questions about the texts. Overall, I expected that the participants holding a dot array in memory while reading would show impaired situation model construction (reduced contradiction effect) in comparison with participants holding a letter string in memory. In this experiment, I also manipulated the difficulty of the dot and letter string tasks. I predicted that because both the dot task and situation model construction needed visuospatial resources, the difficulty of the dot task would affect the readers' ability to comprehend the texts. However, if the letter string task and the generation of a situation model were independent, the difficulty of the letter string task would have little impact on the interpretation of the texts.

\section{EXPERIMENT 2}

\section{Method}

Participants. One hundred and forty students from Gettysburg College took part in this experiment. One student's data were not included in the analyses because that student answered more than half of the comprehension questions that followed each text incorrectly. Of the remaining 139 participants, 70 were in the dot task condition, with 34 in the low-difficulty condition and 36 in the high-difficulty conditions; 69 were in the letter string condition, with 33 in the lowdifficulty condition and 36 in the high-difficulty condition. The participants either received credit for their research participation requirement in their general psychology course or were paid $\$ 5.00$ for their participation.

Design and Materials. This study used a 2 (type of load task: dot task vs. letter string task) $\times 2$ (diff iculty: high vs. low) $\times 2$ (sentence type: consistent sentences vs. inconsistent sentences) mixed design. Type of load task and difficulty of that task were manipulated between subjects, and sentence type was manipulated within subjects. The four dependent variables computed in Experiment 1 were again computed.

Comprehension task. The texts used in Experiment 1 were used also in this experiment. Sentence type was counterbalanced across participants.

Dot task. The visuospatial memory load task was that used by Kruley et al. (1994) and Sims and Hegarty (1997). The dot display was presented on a rectangular grid, 4 cells $\times 4$ cells, that almost filled the computer screen. This display was shown for $667 \mathrm{msec}$. For the low-difficulty dot task condition, three solid black dots were randomly presented within the cells of the grid; for the high-difficulty dot task condition, five solid black dots were randomly presented within the grid. For the verification of the dot display, half of the judgments were same decisions (the grid was identical to the initial 
grid) and half were different decisions (the grid was changed so that one dot from the initial grid was relocated to a randomly determined empty cell adjacent to its original cell). The order of the same/ different trials was random but the same across participants.

Letter string task. This verbal secondary task was used by Sims and Hegarty (1997) and is similar to the verbal interference tasks used in other dual-task studies (Baddeley \& Hitch, 1974). This task was chosen because it has been shown to be equal in difficulty to the dot task (Sims \& Hegarty, 1997). On each trial, a string of letters (all consonants) was presented at the center of the screen for $3 \mathrm{sec}$. For the low-difficulty condition, this was a string of four letters; for the high-difficulty condition, this was a string of six letters. For verification, a single letter was presented at the center of the screen, and the participant had to make a yes/no decision as to whether this letter was part of the original letter string. Half of the trials were true trials and half were false trials; the order of these true/false trials was random but was the same across participants.

Procedure. The participants were randomly assigned to one of the four load type/difficulty conditions (dot task/low difficulty, dot task/high difficulty, letter string task/low difficulty, letter string task/high difficulty) and then one of the two material sets. The participants were told that they would be doing two things in this experiment. Directions informed them, depending on their condition, that they would be briefly presented with a collection of dots on a grid or a string of letters and that they should look at this display/ string carefully because they would have to verify what they had seen. The dot task participants saw the initial display and then later made a "same" or a "different" judgment to a second display. If they saw a display identical to the original display, they pressed the " $\mathrm{S}$ " key for a same judgment (the "B" key covered with a labeled sticker). If they saw a display different from the original display, they pressed the " $\mathrm{D}$ " key for a different judgment (the " $\mathrm{N}$ " key). The letter string participants saw the original string and then later had to verify whether one letter presented was in this original string. If it was in the string, they pressed the "Y" key on the keyboard (the "B" key). If the test letter was not in the original string, they pressed the "N" key. Examples of both true and false trials for both the dot task and the letter string task were presented on paper to the participants, as well as explanations of the correct responses.

The participants were told that between the original display of the dots/letter string and the verification task, they would be receiving short texts to read. The procedure for reading the texts was identical to that in Experiment 1. The participants were given one practice session. When the participants were ready, they began the experimental session that entailed 14 cycles of the procedure above.

\section{Results}

Reading times. As in Experiment 1, the reading times for all sentences of the texts were recorded, with the reading times of the critical target sentences and the posttarget sentences of primary interest. Reading times for critical sentences that were three standard deviations from their mean were discarded. This eliminated approximately $4 \%$ of the data. Results are presented for the critical target sentences when there were no comprehension errors (errors occurred only $7.0 \%$ of the time across all participants).

The reading times for the critical target sentences for all experimental conditions are presented in Table 3 . In an overall 2 (type of load task) $\times 2$ (difficulty of task) $\times 2$ (sentence type) mixed factor ANOVA the triple interaction was not significant $\left[F_{1}(1,135)=2.03, M S_{\mathrm{e}}=64,744\right.$, $\left.p>.10 ; F_{2}(1,52)=1.05, M S_{\mathrm{e}}=51,778, p>.10\right]$. However, the primary interaction of interest, the sentence type $X$
Table 3

Mean Reading Times (in Milliseconds) for Critical Sentences as a Function of Task Type, Sentence Type, and Difficulty: Experiment 2

\begin{tabular}{|c|c|c|c|c|c|}
\hline \multirow[b]{3}{*}{ Task Type } & \multirow[b]{3}{*}{ Difficulty } & \multicolumn{4}{|c|}{ Sentence Type } \\
\hline & & \multicolumn{2}{|c|}{ Consistent } & \multicolumn{2}{|c|}{ Inconsistent } \\
\hline & & $M$ & $S D$ & $M$ & $S D$ \\
\hline \multirow[t]{2}{*}{ Dot } & low & 1,707 & 565 & 1,940 & 589 \\
\hline & high & 1,970 & 635 & 2,114 & 611 \\
\hline \multirow[t]{2}{*}{ Letter string } & low & 1,816 & 494 & 2,170 & 706 \\
\hline & high & 1,569 & 496 & 1,854 & 508 \\
\hline
\end{tabular}

task type interaction, was significant $\left[F_{1}(1,135)=4.57\right.$, $\left.M S_{\mathrm{e}}=64,744 ; F_{2}(1,52)=3.78, M S_{\mathrm{e}}=51,778, p=.07\right]$. These data indicate that in the dot task condition, the contradiction effect (consistent $=1,838 \mathrm{msec}$ vs. inconsistent $=2,027 \mathrm{msec})$, while still significant $\left[F_{1}(1,69)=\right.$ $\left.7.11, M S_{\mathrm{e}}=61,165 ; F_{2}(1,27)=13.16, M S_{\mathrm{e}}=33,647\right]$ was greatly reduced in magnitude in comparison with the letter string task condition (consistent $=1,692 \mathrm{msec}$ vs. inconsistent $=2,012 \mathrm{msec}$ ) $\left[F_{1}(1,68)=51.19, M S_{\mathrm{e}}=\right.$ 68,$\left.112 ; F_{2}(1,27)=13.19, M S_{\mathrm{e}}=68,317\right]$. Furthermore, the difficulty $\times$ sentence type interaction was marginally significant for the dot task $\left[F_{1}(1,68)=3.15, M S_{\mathrm{e}}=\right.$ $\left.61,034, p=.07 ; F_{2}(1,52)=3.64, M S_{\mathrm{e}}=45,306, p=.08\right]$ but was not significant for the letter string task (both $F_{1}$ and $F_{2}<1.0$ ). Planned analyses indicated that for the participants reading with the visuospatial memory load, when the dot task was low in difficulty, the contradiction effect was significant $\left[F_{1}(1,33)=20.35, M S_{\mathrm{e}}=45,562\right.$; $\left.F_{2}(1,13)=8.41, M S_{\mathrm{e}}=45,210\right]$. However, when the dot task was high in difficulty, the contradiction effect was $\operatorname{lost}\left[F_{1}(1,35)=3.23, M S_{\mathrm{e}}=75,621, p>.10 ; F_{2}(1,13)=\right.$ $\left.2.92, M S_{\mathrm{e}}=21,394, p>.10\right]$. For the letter string task, the difficulty manipulation did not affect the contradiction effect. The contradiction effect was strong for both the low- and high-difficulty conditions [for low difficulty $F_{1}(1,32)=22.71, M S_{\mathrm{e}}=91,092 ; F_{2}(1,13)=8.66, M S_{\mathrm{e}}=$ 67,748 , and for high difficulty $F_{1}(1,35)=30.48, M S_{\mathrm{e}}=$ 47,$\left.862 ; F_{2}(1,13)=4.57, M S_{\mathrm{e}}=72,758\right]$.

As in Experiment 1, an analysis that compared participants' average reading time for sentences prior to the critical sentences in each condition was conducted in order to determine whether there were differences in overall comprehension difficulty between conditions. In this analysis, the variables of task type and difficulty were analyzed but not sentence type. This analysis yielded a significant task type $\times$ difficulty interaction $\left[F_{1}(1,133)=\right.$ 6.62, $\left.M S_{\mathrm{e}}=844,147\right]$. Tests for simple effects indicated that the only significant difference between these means was that between the high-difficulty dot task condition $(M=3,449 \mathrm{msec})$ and the high-difficulty letter string condition $(M=2,995 \mathrm{msec})\left[F_{1}(1,70)=4.41, M S_{\mathrm{e}}=\right.$ $841,253]$. When the visuospatial memory load was difficult, readers had more difficulty reading the sentences of the text than when the verbal memory load was difficult.

Performance on dot task/letter string task. The mean percentage of correct responses on the dot and letter string 
Table 4

Mean Percentage Correct Responses as a Function of Task Type, Sentence Type, and Difficulty: Experiment 2

\begin{tabular}{|c|c|c|c|c|c|}
\hline \multirow[b]{3}{*}{ Task Type } & \multirow[b]{3}{*}{ Difficulty } & \multicolumn{4}{|c|}{ Sentence Type } \\
\hline & & \multicolumn{2}{|c|}{ Consistent } & \multicolumn{2}{|c|}{ Inconsistent } \\
\hline & & $M$ & $S D$ & $M$ & $S D$ \\
\hline \multirow[t]{2}{*}{ Dot } & low & 84 & 14 & 87 & 14 \\
\hline & high & 83 & 14 & 79 & 13 \\
\hline \multirow[t]{2}{*}{ Letter string } & low & 85 & 15 & 86 & 15 \\
\hline & high & 76 & 15 & 68 & 16 \\
\hline
\end{tabular}

tasks as a function of difficulty and sentence type is found in Table 4 . An overall 2 (type of load task) $\times 2$ (difficulty of task) $\times 2$ (sentence type) mixed factor ANOVA indicated a significant main effect of task type $\left[F_{1}(1,135)=\right.$ $5.68, M S_{\mathrm{e}}=1.19$ ], with more errors occurring in the letter task than in the dot task. There was also a significant main effect of difficulty $\left[F_{1}(1,135)=20.18, M S_{\mathrm{e}}=1.19\right]$, with more errors occurring in the high-difficulty conditions than in the low-difficulty conditions. Furthermore, there was a significant task type $\times$ difficulty interaction $\left[F_{1}(1,135)=6.21, M S_{\mathrm{e}}=1.19\right]$. Tests for the simple effect of difficulty for each task type indicated that the effect of difficulty was not significant for the dot task (mean percentage correct for low difficulty $=85$, mean percentage for high difficulty $=81)\left[F_{1}(1,68)=2.29, M S_{\mathrm{e}}=\right.$ 1.05], but was significant for the letter string task (mean percentage correct for low difficulty $=85$, mean percentage for high difficulty $=72)\left[F_{1}(1,67)=21.57\right.$, $\left.M S_{\mathrm{e}}=1.33\right]$.

\section{Discussion}

In Experiment 2, I examined the hypothesis that if the construction of a situation model involves perceptual processing-namely, visuospatial processes-then a concurrent task that also engaged these processes would disrupt comprehension more than a concurrent task that did not engage these processes. The results of Experiment 2 supported this prediction. These data show a selective interference effect: Holding a dot array in memory reduced the contradiction effect but holding a letter string in memory did not. Importantly, manipulating the difficulty of the dot task, which had only a small effect on the accuracy of the dot task itself, affected the magnitude of the contradiction effect. In the high-difficulty condition of the dot task, readers failed to show a significant contradiction effect, a reflection of the interfering effects of this concurrent task on situation model construction. On the other hand, manipulating the difficulty of the letter string task, which did affect performance on the letter task itself, did not affect the magnitude of the contradiction effect. Holding a letter string in memory while interpreting a text was difficult, as was evidenced by the performance on the letter string task, but this concurrent task did not interfere with the processes used in generating a situation model of the text.
One might hypothesize that the reason why the contradiction effect was impaired for the readers in the dot task conditions but was not for the readers in the letter string conditions was that the dot task was simply more difficult than the letter string task. Prior research suggests that this is not true (Sims \& Hegarty, 1997), and there was also no evidence for this in the present data. In fact, overall performance on the dot task was better than performance on the letter string task. In addition, there was no evidence for any clear speed/accuracy tradeoff in these data; in the dot task and the letter string task, there was an inconsistent relationship between reading times and performance on the concurrent task. In the dot task, slower reading times in the high-difficulty condition were associated with poorer performance on the dot task. This suggests that the two tasks were sharing resources and were interfering with one another. In the letter string task, faster reading times in the high-difficulty condition were accompanied by poorer performance on the task itself. One explanation for the fast reading times in the highdifficulty letter string condition is that readers knew that when given a six-letter string to hold in memory, they were not going to do well on the task unless they read the sentences of the text quickly. Despite this strategy, however, they had difficulty with the letter decision. Furthermore, although these letter string participants read the texts quickly, they still showed evidence of building situation models - the contradiction effect was very strong in this condition. This suggests that the letter string task and the comprehension task were independent of one another.

\section{CONCLUSIONS}

Researchers have claimed that situation models may not be propositional as are their complementary representations, the surface structure and the textbase, but instead may be image-like or have perceptual components (Ericsson \& Kintsch, 1995; Glenberg, Meyer, \& Lindem, 1987; Johnson-Laird, 1983). Recently, the argument has been made that comprehension entails indexing words to objects that are represented as perceptual symbols stored in memory. These perceptual representations determine affordances - or a way for the reader to envision the interaction of these objects that the text is describing (Barsalou, 1999; Glenberg \& Robertson, 1999). The present research tested the hypothesis that situation model construction involves perceptual processes-namely, visuospatial processes - and that therefore, if those processes are engaged in another task during reading, comprehension suffers. In Experiment 1, holding high-imagery sentences in memory prevented readers from constructing a situation model, as was reflected in the absence of a significant contradiction effect, but holding low-imagery sentences in memory did not affect the contradiction effect. Experiment 2 indicated that a difficult visuospatial memory load interfered more with the comprehension task than did a verbal memory load. These results support the con- 
tention that text comprehension involves constructing situation models that have perceptual components.

It is interesting to note that the evidence of situation model construction, the contradiction effect, was never completely eliminated in either Experiment 1 or Experiment 2. It was reduced when there were demands on visuospatial resources, indicating interference, but even with those demands, readers could interpret the text enough to show a trend toward a contradiction effect. It may be that maintaining a visual array does not completely deplete visuospatial resources and, therefore, that some are available for comprehension processes. For good readers, the processes involved in situation model construction may be fairly well automatized, thus requiring few resources. Therefore, only a concurrent task that requires heavy use of visuospatial processes could disrupt situation model construction, and this is perhaps why the high-difficulty dot task condition showed the greatest interference effect. Another possibility for why the contradiction effect appears resistant to interference is that when visuospatial resources are being used, perhaps an alternative nonvisual language process is employed to generate a situation model. This alternative method of construction may provide a limited interpretation of the text.

The present research does suggest that visuospatial processes are engaged during situation model construction, but these data do not necessarily indicate that situation models are image-like. Many situation model researchers have argued that it is unrealistic to believe that readers construct "lifelike" mental representations (McKoon \& Ratcliff, 1992; Zwaan \& Radvansky, 1998). Generating a detailed mental image of a situation described by a text would require much time and effort, and this would be inefficient for a level of representation that needs to be fluid and can change with each new unit of text (Denis, 1996). However, creating a representation that uses perceptual symbols to represent certain protagonists or objects, or to instantiate inferences generated by the reader, is consistent with the nature of situation models. It is also possible that perceptual processes, or visuospatial processes, are used in constructing a situation model that is ultimately propositional in nature.

\section{REFERENCES}

Albrecht, J. E., \& O'Brien, E. J. (1993). Updating a mental model: Maintaining both local and global coherence. Journal of Experimental Psychology: Learning, Memory, \& Cognition, 19, 1061-1070.

BADDELEY, A. D. (1992). Is working memory working? The Fifteenth Bartlett Lecture. Quarterly Journal of Experimental Psychology, 44A, $1-31$.

Baddeley, A. D., \& Hitch, G. (1974). Working memory. In G. H. Bower (Ed.), The psychology of learning and motivation (Vol. 8, pp. 47-89). New York: Academic Press.

Barsalou, L. W. (1999). Perceptual symbol systems. Behavioral \& Brain Sciences, 22, 577-660.

BrooKs, L. R. (1968). Spatial and verbal components of the act of recall. Canadian Journal of Psychology, 22, 349-368.
Denis, M. (1996). Imagery and the description of spatial configurations. In M. deVega, M. J. Intons-Peterson, P. N. Johnson-Laird, M. Denis, \& M. Marschark (Eds.), Models of visuospatial cognition (pp. 128-197). New York: Oxford University Press.

ERICSSON, K. A., \& KINTSCH, W. (1995). Long-term working memory. Psychological Review, 102, 211-245.

Glass, A. L., EdDy, J. K., \& Schwanenflugel, P. J. (1980). The verification of high and low imagery sentences. Journal of Experimental Psychology: Human Learning \& Memory, 6, 692-704.

Glass, A. L., Millen, D. R, Beck, L. G., \& EdDY, J. K. (1985). Representation of images in sentence verification. Journal of Memory \& Language, 24, 442-465.

Glenberg, A. M. (1997). What is memory for. Behavioral \& Brain Sciences, 20, 1-55.

Glenberg, A. M., \& Langston, W. E. (1992). Comprehension of illustrated text: Pictures help to build mental models. Journal of Memory \& Language, 31, 129-151.

Glenberg, A. M., Meyer, M., \& Lindem, K. (1987). Mental models contribute to foregrounding during text comprehension. Journal of Memory \& Language, 26, 69-83.

Glenberg, A. M., \& Robertson, D. A. (1999). Indexical understanding of instructions. Discourse Processes, 28, 1-26.

Huitema, J., Dopkins, S. E., Kinn, C. M., \& Myers, J. L. (1993). Connecting goals and actions during reading. Journal of Experimental Psychology: Learning, Memory, \& Cognition, 19, 1053-1060.

JOHNSON-LAIRD, P. N. (1983). Mental models: Towards a cognitive science of language, inference, and consciousness. Cambridge, MA: Harvard University Press.

KInTSCH, W. (1998). Comprehension: A paradigm for cognition. Cambridge: Cambridge University Press.

KintsCH, W., \& WeLSCH, D. M. (1991). The construction-integration model: A framework for studying memory for text. In W. E. Hockley \& S. Lewandowsky (Eds.), Relating theory and data: Essays in human memory in honor of Bennett B. Murdock (pp. 367-385). Hillsdale, NJ: Erlbaum.

Kruley, P., Sciama, S. C., \& Glenberg, A. M. (1994). On-line processing of textual illustrations in the visuospatial sketchpad: Evidence from dual-task studies. Memory \& Cognition, 22, 261-272.

MacWhinney, B. (1999). The emergence of language from embodiment. In B. MacWhinney (Ed.), The emergence of language (pp. 213256). Mahwah, NJ: Erlbaum.

McKoon, G., \& Ratcliff, R. (1992). Inference during reading. Psychological Review, 99, 440-466.

Morrow, D. G. (1994). Spatial models created from text. In H. van Oostendorp \& R. A. Zwaan (Eds.), Naturalistic text comprehension (pp. 57-78). Norwood, NJ: Ablex.

Myers, J. L., \& O'Brien, E. J. (1998). Accessing the discourse representation during reading. Discourse Processes, 26, 131-157.

Myers, J. L., O'Brien, E. J., Albrecht, J. E., \& Mason, R. A. (1994). Maintaining global coherence during reading. Journal of Experimental Psychology: Learning, Memory, \& Cognition, 20, 876-886.

O'Brien, E. J., \& Albrecht, J. E. (1992). Comprehension strategies in the development of mental model. Journal of Experimental Psychology: Learning, Memory, \& Cognition, 18, 777-784.

Paivio, A. (1969). Mental imagery in associative learning and memory. Psychological Review, 76, 241-263.

Sims, V. K., \& Hegarty, M. (1997). Mental animation in the visuospatial sketchpad: Evidence from dual-task studies. Memory \& Cognition, 25, 321-332.

VAN DiJK, T. A., \& KinTsch, W. (1983). Strategies in discourse comprehension. New York: Academic Press.

ZWAAN, R. A. (1999). Embodied cognition, perceptual symbols, and situation models. Discourse Processes, 28, 81-88.

Zwaan, R. A., Langston, M. C., \& Graesser, A. C. (1995). The construction of situation models in narrative comprehension: An eventindexing model. Psychological Science, 6, 292-297.

ZWAAN, R A., \& RADVANSKY, G. A. (1998). Situation models in language comprehension and memory. Psychological Bulletin, 123, 162-185. 
APPENDIX

Example Passage From Experiments 1 and 2

\section{Introduction:}

Today, Mary was meeting a friend for lunch. She arrived early at the restaurant and decided to get a table. After she sat down, she started looking at the menu.

\section{Elaboration:}

Consistent: This was Mary's favorite restaurant because it had fantastic junk food. Mary enjoyed eating anything that was quick and easy to fix. In fact, she ate at McDonalds at least three times a week. Mary never worried about her diet and saw no reason to eat nutritious foods.

Inconsistent: This was Mary's favorite restaurant because it had fantastic health food. Mary, a health nut, has been a strict vegetarian for ten years. Her favorite food was cauliflower. Mary was so serious about her diet that she refused to eat anything which was fried or cooked in grease.

\section{Background:}

After about ten minutes, Mary's friend arrived. It had been a few months since they had seen each other. Because of this they had a lot to talk about and chatted for over a half hour. Finally, Mary signaled the waiter to come take their orders. Mary checked the menu one more time. She had a hard time deciding what to have for lunch.

\section{Critical Target Sentence:}

Mary ordered a cheeseburgerand fries.

\section{Posttarget Sentence:}

She handed the menu back to the waiter.

\section{Conclusion:}

Her friend didn't have as much trouble deciding what she wanted. She ordered and they began to chat again. They didn't realize there was so much for them to catch up on. 